\title{
Sleep fragmentation and intermittent hypoxemia are associated with decreased insulin sensitivity in obese adolescent Latino males
}

\author{
Daniel J. Lesser ${ }^{1}$, Rajeev Bhatia ${ }^{1}$, Winston H. Tran², Flavia Oliveira², Ricardo Ortega' ${ }^{1}$, Thomas G. Keens ${ }^{1}$, Steven D. Mittelman³ , \\ Michael C.K. Khoo ${ }^{2}$ and Sally L. Davidson Ward ${ }^{1}$
}

BACKGROUND: Although sleep-related breathing disorder (SRBD) has been linked to insulin resistance in adults, this has not been as well established in children. We hypothesized that the severity of SRBD in adolescents was associated with metabolic impairment.

METHODS: Polysomnography was performed on obese, Latino males referred for snoring. The frequently sampled intravenous glucose tolerance test was used to assess glucose homeostasis. Total-body dual-energy X-ray absorptiometry was used to quantify adiposity.

RESULTS: A total of 22 males (mean age \pm SD: $13.4 \pm 2.1 \mathrm{y}$, BMI z-score $2.4 \pm 0.3$, obstructive apnea hypopnea index $4.1 \pm$ 3.2) were studied. After correcting for age and adiposity in multiple-regression models, Log frequency of desaturation (defined as $\geq 3 \%$ drop in oxygen saturation from baseline) negatively correlated with insulin sensitivity. Sleep efficiency was positively correlated with glucose effectiveness $\left(S_{G}\right.$, the capacity of glucose to mediate its own disposal). The Log total arousal index was positively correlated with Log homeostasis model assessment-estimated insulin resistance.

CONCLUSION: Sleep fragmentation and intermittent hypoxemia are associated with metabolic impairment in obese adolescent Latino males independent of age and adiposity. We speculate that SRBD potentiates the risk for development of metabolic syndrome and type 2 diabetes in the obese adolescent population.

hildhood obesity remains a critical health issue associated with long-term consequences worldwide (1). The early stages of metabolic dysfunction, as it pertains to children, have not been wholly identified. Sleep-related breathing disorder (SRBD) has a high prevalence in obese individuals and has been linked to insulin resistance in the adult population (2-4). In addition, SRBD likely contributes to the pathophysiology of the metabolic syndrome (5). Although the mechanisms linking SRBD to the metabolic syndrome are not fully known, intermittent hypoxemia, altered inflammatory pathways, and dysregulation of the autonomic nervous system have been hypothesized $(3,6)$.

Even less is known about the relationship between SRBD and disorders of glucose metabolism in the pediatric population. Although some studies have found that the severity of obstructive sleep apnea syndrome independently associates with insulin resistance $(7,8)$, others have found obesity to be the major determinant $(9,10)$. This study includes data only from obese adolescent Latin males. Latino youth are a group with a high risk for development of type 2 diabetes mellitus and other complications of the metabolic syndrome (11). A key rationale for limiting the sex and ethnicity of the study population was to reduce the number of potential confounding variables, as insulin resistance and the insulin response to glucose are known to vary with ethnicity and gender (12).

To evaluate glucose metabolism, the majority of previous studies relating SRBD to insulin resistance have used static measures, such as the homeostasis model assessmentestimated insulin resistance $\left(\mathrm{HOMA}_{\mathrm{IR}}\right)$, based on fasting blood glucose and insulin levels $(4,13)$. However, the pathophysiology of type 2 diabetes involves not only insulin resistance, but also impairment in the compensatory response via pancreatic $\beta$-cell function $(4,14)$. Therefore, it would be preferable to assess glucose metabolism considering both insulin sensitivity $\left(S_{\mathrm{I}}\right)$ and pancreatic $\beta$-cell activity (4). The frequently sampled intravenous glucose tolerance test (FSIVGTT) allows simultaneous assessment of $S_{I}$ and pancreatic $\beta$-cell function through the use of a mathematical model such as the Bergman minimal model (15).

The aim of this study was to use the FSIVGTT to describe the relationship between glucose-insulin physiology and SRBD in a group of obese adolescent Latino males. We hypothesized that the measures of SRBD severity (such as the desaturation index (DESAT) and the obstructive apnea hypopnea index (OAHI) ) would be associated with measures of impaired glucose metabolism derived from FSIVGTT, and that these associations would be independent of the degree of obesity.

\footnotetext{
The first two authors contributed equally to this work.

'Division of Pediatric Pulmonology, Children's Hospital Los Angeles, University of Southern California Keck School of Medicine, Los Angeles, California; ${ }^{2}$ University of Southern California Vitterbi School of Biomedical Engineering, Los Angeles, California; ${ }^{3}$ Center for Endocrinology, Diabetes and Metabolism, Children's Hospital Los Angeles, University of Southern California Keck School of Medicine, Los Angeles, California. Correspondence: Sally L. Davidson Ward (sward@chla.usc.edu)
} 


\section{RESULTS}

A total of 22 obese Latino males with a mean age of $13.2 \pm$ 2.1 y completed the study. The average duration between the polysomnography and FSIVGTT was $72.1 \pm 67.8 \mathrm{~d}$. None of the subjects received any treatment for SRBD between these two components of the study. All subjects had BMI $\geq 95$ th percentile for age/gender and the mean BMI z-score was 2.4 \pm 0.3 (Table 1). Polysomnography results revealed a mean OAHI of $4.1 \pm 3.2$ events/h with a range of 1.0-14.1. On average, the subjects had $9.8 \pm 11.1$ desaturation events/h of sleep (Table 1). Assuming a cutoff OAHI of 5 events/h, we classified 16 of the subjects as having no or mild SRBD (OAHI $<5)$, with the other 6 having moderate to severe SRBD (OAHI $\geq 5$ ). Table 1 also shows considerable variability in the metabolic parameters across subjects. Please refer to the Methods section for a description of the parameters measured by the

Table 1. Demographic, sleep, and metabolic characteristics

\begin{tabular}{|c|c|c|}
\hline Variable, $n=22$ & Mean \pm SD & Range \\
\hline \multicolumn{3}{|l|}{ Demographic } \\
\hline Age (y) & $13.4 \pm 2.1$ & $10.3-17.9$ \\
\hline $\mathrm{BMI}\left(\mathrm{kg} / \mathrm{m}^{2}\right)$ & $34.4 \pm 6.3$ & $25.3-52.5$ \\
\hline BMI (z-score) & $2.4 \pm 0.3$ & $2.0-3.2$ \\
\hline Total body fat (g) & $35,869 \pm 12,113$ & $18,858-63,610$ \\
\hline Total percentage body fat & $41 \pm 5.0$ & $29.9-50.0$ \\
\hline Trunk fat (g) & $16,347 \pm 4,996$ & $8,871-25,914$ \\
\hline Percentage trunk fat & $40.8 \pm 4.4$ & $30.9-46.0$ \\
\hline \multicolumn{3}{|l|}{ Polysomnography } \\
\hline Total sleep time (min) & $316 \pm 68.6$ & $163-444$ \\
\hline Sleep efficiency (\%) & $80.6 \pm 10.4$ & $55-97$ \\
\hline REM (\%TST) & $14.5 \pm 7.7$ & $0-30$ \\
\hline REM latency (min) & $112.5 \pm 63.5$ & $0-231$ \\
\hline Obstructive AHI (n/h) & $4.1 \pm 3.2$ & $1.0-14.1$ \\
\hline Desaturation index $(n / h)$ & $9.8 \pm 11.1$ & $0.0-40.1$ \\
\hline Total arousal index $(n / h)$ & $11.7 \pm 6.6$ & $4.1-29.0$ \\
\hline $\mathrm{S}_{\mathrm{p}} \mathrm{O}_{2}, \operatorname{nadir}(\%)$ & $87 \pm 4.6$ & $79-94$ \\
\hline Mean $\mathrm{S}_{\mathrm{p}} \mathrm{O}_{2}(\%)$ & $95 \pm 1.8$ & $91-97$ \\
\hline \multicolumn{3}{|l|}{ Metabolic } \\
\hline$S_{1}((\mathrm{mU} / \mathrm{l}) / \mathrm{min})$ & $4.1 \pm 4.8$ & $0.9-22.2$ \\
\hline $\operatorname{AIRg}(\mathrm{mU} / \mathrm{l}) \min$ & $1,003 \pm 796$ & $148-3,360$ \\
\hline $\mathrm{DI}\left(S_{1} \times \mathrm{AIRg}\right)$ & $2,548 \pm 1,759$ & $537-8,328$ \\
\hline$S_{G}\left(\times 1 / 10^{2} / \mathrm{min}\right)$ & $0.019 \pm 0.009$ & $0.003-0.039$ \\
\hline Fasting glucose(mg/dl) & $84.7 \pm 7.4$ & $70.5-100.0$ \\
\hline Fasting insulin $(\mu \mathrm{U} / \mathrm{ml})$ & $13.8 \pm 8.7$ & $2.0-39.5$ \\
\hline $\mathrm{HOMA}_{\mathrm{IR}}$ & $2.97 \pm 2.06$ & $0.40-9.51$ \\
\hline FGIR & $9.63 \pm 8.5$ & $2.5-40.8$ \\
\hline QUICKI & $0.15 \pm 0.02$ & $0.12-0.20$ \\
\hline
\end{tabular}

$\mathrm{AHI}$, apnea hypopnea index; AIRg, acute insulin response; DI, disposition index; FGIR, fasting glucose/insulin ratio; $\mathrm{HOMA}_{\mathbb{R}^{\prime}}$, homeostasis model assessment-estimated insulin resistance; QUICKI, quantitative insulin sensitivity check index; REM, rapid eye movement; $S_{G^{\prime}}$ glucose effectiveness; $S_{1}$, insulin sensitivity; $S_{\mathrm{p}} \mathrm{O}_{2}$, oxy-hemoglobin saturation; TST, total sleep time.
FSIVGTT. The ranges in fasting serum and FSIVGTT values displayed in Table 1 are consistent with those reported previously for obese Latino children in the Greater Los Angeles area (12). As expected, the static measures of insulin resistance correlated with those of the FSIVGTT. As $S_{\mathrm{I}}$ increased, HOMA decreased $(r=-0.782, P<0.001)$ (Figure 1$)$ whereas the quantitative $\mathrm{S}_{\mathrm{I}}$ check index increased $(r=0.787, P=0.000)$.

The Pearson coefficients of the pairwise correlations between the raw values of the sleep-related and metabolic parameters, displayed in Table 2, show that a number of pairs of these parameters were significantly correlated. Of these, the strongest correlations were between Log DESAT and Log $S_{\mathrm{I}}(r=$ -0.53 ) and between Log total arousal index (TAI) and Log fasting insulin $(r=0.56)$.

A crucial aim of the study was to use multiple linear regression to assess the impact of SRBD on glucose homeostasis independent of age and obesity. BMI and regional fat distribution (as measured by dual-energy X-ray absorptiometry (DEXA)) were chosen as measures to correct for adiposity. Because Tanner staging was not available in all patients, age (which strongly correlated with Tanner stage in the 16 subjects available, $r=0.57, P=0.021$, Spearman rank correlation) was used as a surrogate marker for pubertal stage.

After correcting for age and adiposity (as measured by BMI z-score, DEXA percentage body fat and DEXA percentage trunk fat) using multiple linear regression, we found that some of the correlation coefficients that appeared significant in Table 2 were no longer statistically significant. However, the metabolic-sleep pairs with the strongest correlations remained significant (Table 3$)$. In particular, Log(DESAT) remained moderately correlated with $\log \left(S_{\mathrm{I}}\right)$ even after taking into account the confounding effects of age and BMI or fat distribution/amount measured by DEXA (Figure 2). Log(TAI) remained positively correlated with $\log ($ fasting insulin) (Figure 3$)$. $\log (\mathrm{TAI})$ also correlated with $\log \left(\mathrm{HOMA}_{\mathrm{IR}}\right)$ when DEXA, but not BMI

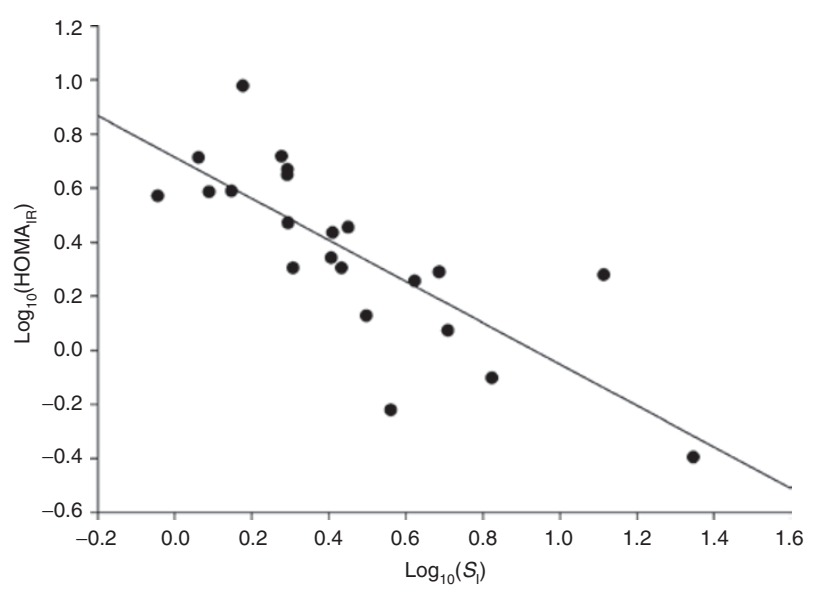

Figure 1. Homeostasis model assessment-estimated insulin resistance $\left(\mathrm{HOMA}_{\mathbb{R}}\right)$ negatively correlates with insulin sensitivity. Scatter plot shows significant negative correlation between the conventional static index of insulin resistance based on fasting serum measurements $\left(\mathrm{HOMA}_{\mathbb{R}}\right)$ and insulin sensitivity $\left(S_{1}\right)$, a parameter derived from the frequently sampled intravenous glucose tolerance test. Pearson correlation coefficient $r=$ $-0.78, P=0.000017$. 
Table 2. Pairwise correlations ( $r$ value) between key polysomnographic and metabolic parameters (before adjustment for age and adiposity)

\begin{tabular}{|c|c|c|c|c|c|c|}
\hline & Total sleep time & Efficiency (\%) & Log TAI & $\mathrm{S}_{\mathrm{p}} \mathrm{O}_{2}$ nadir & Log DESAT index & Log OAHI \\
\hline $\log S_{1}$ & -0.11 & -0.15 & -0.42 & $0.443^{*}$ & $-0.53^{*}$ & -0.29 \\
\hline Log AIRg & 0.39 & 0.32 & 0.31 & -0.329 & 0.38 & 0.14 \\
\hline$S_{G}$ & 0.17 & $0.44^{*}$ & -0.10 & 0.160 & -0.28 & -0.060 \\
\hline Log Fast Ins & 0.032 & -0.049 & $0.56^{* *}$ & -0.325 & 0.38 & 0.23 \\
\hline Log $\mathrm{HOMA}_{\mathrm{IR}}$ & 0.024 & -0.065 & $0.55^{*}$ & -0.343 & 0.38 & 0.22 \\
\hline
\end{tabular}

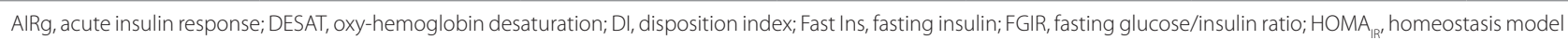

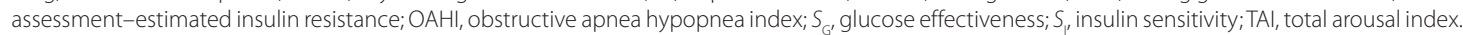

${ }^{*} P \leq 0.05 ;{ }^{*} P \leq 0.01$.

Table 3. Metabolic vs. sleep parameters after adjustment for age and adiposity

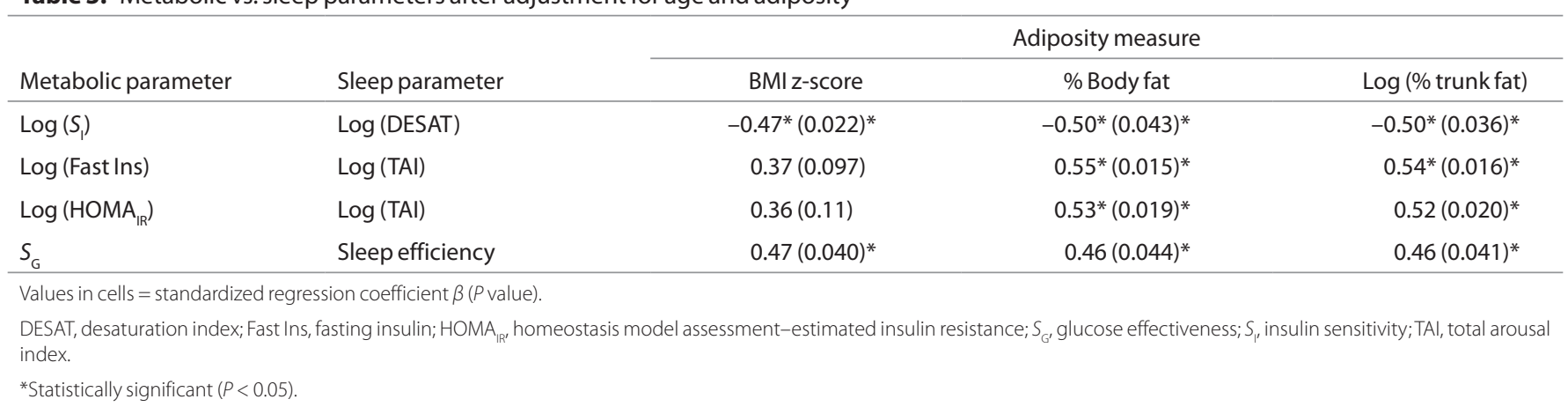

Z-score, was used to measure the degree of obesity (Table 3). A somewhat unexpected finding was the correlation between sleep efficiency and glucose effectiveness $\left(S_{\mathrm{G}}\right)$ (Figure 4$)$. Thus, sleep fragmentation and intermittent hypoxemia were found to be associated with metabolic impairment in this group of patients independent of age and adiposity.

\section{DISCUSSION}

This study evaluated the association between SRBD and the factors characterizing glucose-insulin metabolism in obese adolescent Latino males using the FSIVGTT. We found that intermittent hypoxemia correlates negatively with $S_{\mathrm{I}}$. Sleep efficiency correlates positively with $S_{G}$, the capacity of glucose to mediate its own disposal. We also found that the degree of sleep disruption as reflected by $\log (\mathrm{TAI})$ was positively correlated with $\log \left(\mathrm{HOMA}_{\mathrm{IR}}\right.$ ) (a marker of insulin resistance based on fasting measurements). Furthermore, all of these associations were independent of age and adiposity.

The strength of our study centers on the use of the FSIVGTT to measure glucose metabolism, allowing simultaneous estimation of both insulin resistance and pancreatic $\beta$-cell action. By using the FSIVGTT to measure glucose metabolism, we identified in children a relationship between SRBD and pancreatic $\beta$-cell responsiveness as well as insulin sensitivity (disposition index), both of which are fundamental to understanding the pathophysiology leading to type 2 diabetes (14). The use of DEXA allowed us to more accurately quantify and adjust statistically for differences in adiposity among the subjects than would the use of BMI alone. Furthermore, by restricting the pool of subjects to only adolescent Latino males, we were able to minimize the potential confounding effects of gender, ethnicity, and age.

Whether SRBD imposes an added risk to obesity for the development of pediatric insulin resistance remains controversial $(7,9,16-22)$. The majority of studies considering this issue used static measurements of insulin and glucose as markers of insulin resistance in contrast to our methodology, which employed the FSIVGTT. Although we identified a correlation between $\log$ DESAT and $\log S_{\mathrm{I}}$, an association between Log DESAT and each of the static measures of insulin resistance was not identified, particularly after the dependent and independent variables were adjusted for age and adiposity. This suggests that the measures of insulin resistance based on fasting serum values in relation to SRBD are not as accurate as FSIVGTT in measuring markers of early metabolic dysfunction.

We observed that subjects with lower sleep efficiency had lower $S_{\mathrm{G}}$ and that increased arousals correlated with increased fasting insulin and $\mathrm{HOMA}_{\mathrm{IR}}$. This suggests the role of sleep fragmentation in the pathogenesis of insulin resistance in pediatric patients. In adults, it has been shown that fragmentation of sleep across all stages is associated with a decrease in $S_{\mathrm{I}}$ and $S_{\mathrm{G}}(23)$. What is the mechanism explaining the link between sleep fragmentation and metabolic dysfunction? Sleep fragmentation may abnormally activate the hypothalamic pituitary axis $(24,25)$. This activation may then result in glucocorticoid inhibition of insulin secretion and insulin resistance via modification of multiple insulin-mediated glucose transport systems $(26,27)$.

Our study found the frequency of oxygen desaturation episodes, defined as only a $\geq 3 \%$ drop in oxy-hemoglobin 


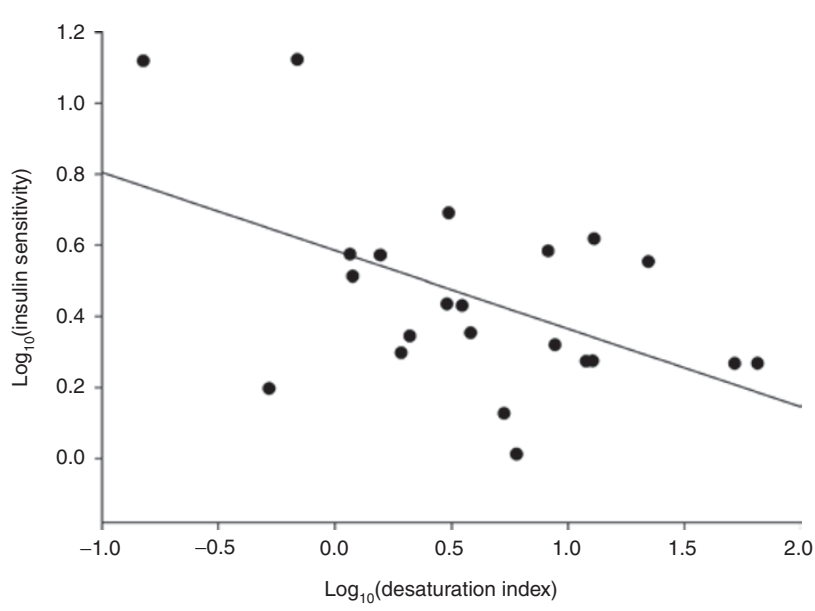

Figure 2. Log (insulin sensitivity) was linearly and negatively correlated with Log (desaturation index), following adjustment in both independent and dependent variables for age and adiposity (BMI z-score, total percentage fat, and percentage trunk fat). For BMI z-score as the adiposity measure, $\beta=-0.47(P=0.022)$.

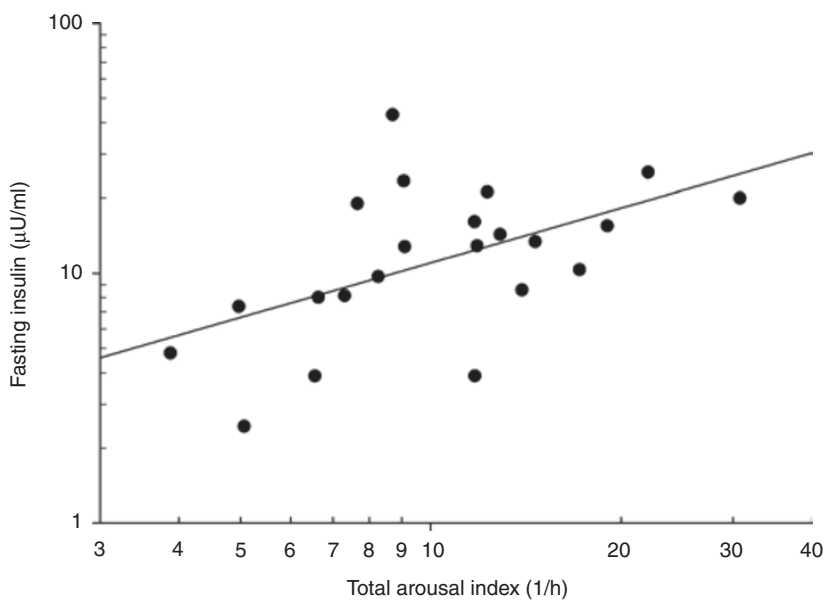

Figure 3. Sleep fragmentation correlates with increased fasting insulin levels. Log plot between fasting insulin and total arousal index (TAI) showing linear and positive correlation, independent of age and adiposity (total $\%$ body fat and trunk $\%$ fat). For total $\%$ body fat as the adiposity measure, $\beta=0.55(P=0.015)$.

saturation $\left(\mathrm{S}_{\mathrm{p}} \mathrm{O}_{2}\right)$ from baseline, to be associated with lower insulin sensitivity. This finding suggests that even mild episodes of frequent oxygen desaturation can be associated with altered insulin-glucose physiology in susceptible pediatric populations. Although the DESAT negatively correlates with insulin sensitivity, it is not correlated with $\beta$-cell responsiveness (acute insulin response). To maintain normal glucose homeostasis, a decline in peripheral insulin sensitivity is expected to be compensated for by an increase in pancreatic $\beta$-cell responsiveness. However, our study did not identify the expected increase in $\beta$-cell responsiveness with increasing frequency of desaturation, suggesting dysfunction of pancreatic $\beta$-cells. We speculate that the degree of hypoxemia during sleep in obese pediatric patients impacts both peripheral insulin resistance and pancreatic $\beta$-cell action (4).

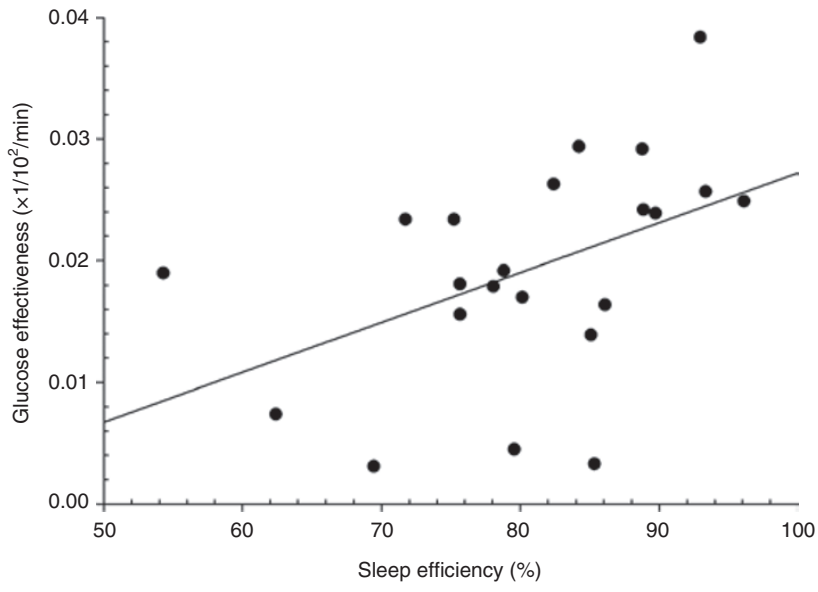

Figure 4. Glucose effectiveness $\left(S_{G}\right)$ was linearly and positively correlated with sleep efficiency, following adjustment in both independent and dependent variables for age and adiposity (BMI z-score, total percentage body fat, and percentage trunk fat). For BMI $z$-score, $\beta=0.47$ $(P=0.040)$.

A number of unique features and limitations of this study merit discussion. Because our study was cross-sectional, it is not possible to arrive at any definitive conclusions about causal mechanisms from our finding of an association between $S_{\mathrm{I}}$ and intermittent hypoxemia. A longitudinal study involving data posttreatment might provide stronger support to our hypothesis. However, taken together with the findings from other studies, the mechanism by which SRBD contributes to insulin resistance most likely centers on intermittent episodes of oxygen desaturation, leading to alterations in key physiologic pathways. Hypoxia has been linked to disturbances in inflammatory pathways manifested by alterations in proinflammatory cytokines and abnormal transcription pathways that have been postulated to impact development of the metabolic syndrome (3). Furthermore, animal models have suggested that intermittent hypoxia leads to insulin resistance even in lean mice (28). Although inflammatory markers were not measured in this study, alterations in inflammatory pathways caused by SRBD likely contributed to its association with insulin resistance.

An additional limitation of our study relates to the incomplete Tanner stage information of our subjects, which occurred due to some subjects' preference to not participate in this portion of the exam. Although it is likely that the stage of puberty affected glucose-insulin dynamics $(29,30)$, we found a strong correlation between age and Tanner stage in the subjects for whom we were able to obtain Tanner stage data. Thus, age can be viewed as a surrogate marker of pubertal development in our subjects. Even after adjusting for age and adiposity, sleep fragmentation and intermittent hypoxemia were found to be associated with metabolic impairment. Further longitudinal studies are indicated to explore how puberty effects the association between metabolic impairment and SRBD in obese children.

Another potential confounder of our results is the fact that the FSIVGTT and polysomnography components of the study were carried out separately for each subject. However, none of the subjects received therapy for SRBD or insulin 
resistance in the time between the two parts of the research protocol. Thus, the time spent between the two portions of the study seems unlikely to have impacted the results. To avoid potentially confounding effects of therapies, we chose to exclude diabetic patients and those on antihypertensive medications. This potentially excluded a clinically relevant segment of pediatric patients at high risk for incurring complications of obesity. However, the majority of patients referred to our sleep center are nondiabetic and do not use medications for hypertension. Finally, our initial statistical method applied pairwise correlations across a number of variables (Table 2), which may lead to errors in inference. The pairwise correlation was used to screen for potentially significant associations. Subsequently, we used multiple linear regression to determine the correlations upon which the conclusions are based.

In summary, sleep fragmentation and intermittent hypoxemia in obese adolescent Latino males is associated with impairment in insulin sensitivity and $S_{G}$ independent of age and adiposity. These findings are critical considering the high prevalence of obesity, SRBD, and metabolic impairment in children. It would be important to continue to explore whether treatment of SRBD at an early age (e.g., positive pressure therapy, weight loss) can improve metabolic function and decrease the potential comorbidities in obese children.

\section{METHODS}

\section{Subjects}

Twenty-two obese (BMI $\geq 95$ th percentile for age/gender), Latino males $10-17$ y old were recruited from patients referred to our center for snoring. Exclusion criteria included a history of lung disease other than mild asthma, type 2 diabetes mellitus, hypertension requiring the use of antihypertensive medications, or use of stimulant medications for attention deficit hyperactivity disorder. In addition, subjects using positive airway pressure therapy or supplemental oxygen were excluded. The Children's Hospital Los Angeles Institutional Review Board approved the study, and written informed consent/assent was obtained.

\section{Measurement of Body Fat}

Each subject's weight to the nearest $0.1 \mathrm{~kg}$ was measured with a digital scale and height to the nearest centimeter was measured with a wallmounted stadiometer. A physician determined Tanner pubertal stage on subjects who assented to the exam. BMI was calculated as weight/ height ${ }^{2}$, compared with standard reference values, and expressed as a percentile for age/gender and z- score based on the Centers for Disease Control and Prevention growth charts. Total body fat mass, trunk fat mass, percentage body fat, and percentage trunk fat were determined from DEXA using a Hologic QDR-4500 Delphi W (Hologic, Bedford, MA) scanner.

\section{Indexes of Metabolic Function}

After overnight fasting, subjects were tested in a general clinical research center with the standard FSIVGTT in the morning. Fasting glucose and insulin levels were expressed as the mean of two samples collected 15 and $5 \mathrm{~min}$ before time zero. At time zero, glucose ( $25 \%$ dextrose, $0.3 \mathrm{~g} / \mathrm{kg}$ body weight) was administered intravenously over $1-2 \mathrm{~min}$. At $\mathrm{t}=20 \mathrm{~min}$, insulin $(0.02 \mathrm{U} / \mathrm{kg}$ body weight, Humulin Regular insulin; Eli Lilly, Indianapolis, IN) was injected. Blood samples were collected at: $2,4,8,12,16,19,22,24,26,30,35$, $40,50,70,100,140$, and $180 \mathrm{~min}$ and centrifuged $(7 \mathrm{~min}, 3,500 \mathrm{rpm}$, $4{ }^{\circ} \mathrm{C}$ ) to obtain plasma. Aliquots were frozen at $-20^{\circ} \mathrm{C}$. Glucose was assayed using the glucose oxidase method (Vitros 5.1, Orthoclinical Diagnostics; Johnson and Johnson, Rochester, NY). Insulin was assayed in duplicate by using a two-site immunochemiluminometric assay (Esoterix, Calabasas Hills, CA).

The Bergman minimal model was applied to determine insulinglucose physiology (Minmod Millenium 2004 computer program, version 6.02; Minmod, Richard N. Bergman, USC) (15,31-33). This well-accepted method utilizes compartmental modeling of the pattern of glucose and insulin concentrations in the blood to quantify the ability of the pancreas to secrete insulin in response to a glucose load (acute insulin response), the ability of insulin to enhance the disposal of glucose (insulin sensitivity, $S_{\mathrm{I}}$ ), and the ability of glucose to enhance its own disposal from the plasma pool $\left(S_{G}\right)$. Because the amount of insulin secretion necessary to maintain glucose homeostasis depends on insulin sensitivity, it is important to quantify beta cell function with respect to insulin sensitivity. Therefore, the minimal model facilitates the calculation of the disposition index, which represents the ability of the pancreas to compensate for decreases in insulin sensitivity. Three other indexes of insulin resistance based on the subject's fasting measurements were derived: (i) homeostatic model assessment of insulin resistance $\left(\mathrm{HOMA}_{\mathrm{IR}}\right.$; fasting insulin $(\mu \mathrm{U} / \mathrm{ml}) \times$ fasting glucose (mg/dl)/405), (ii) quantitative insulin sensitivity check index, defined as $1 /$ (Log fasting insulin + Log fasting glucose) (34); and (iii) fasting glucose/insulin ratio.

\section{Polysomnography}

Overnight polysomnography was performed on all subjects. Surface electrodes were connected to subjects to monitor electrocardiogram, chin electromyogram, electroencephalogram (frontal, central, and occipital), and electro-oculogram. Respiratory inductance plethysmographies of the chest and abdomen and pulse oximetry were recorded. A nasal-oral thermistor was used to evaluate combined airflow. Studies were scored by two investigators (blinded to the results of the FSIVGTT) arriving at consensus. Raw data were scored according to criteria established by the American Academy of Sleep Medicine $(35,36)$. The OAHI was calculated based on the number of obstructive apneas, hypopneas, and mixed apneas per hour of sleep. The TAI (spontaneous, movement, or respiratory) was calculated based on the total number of arousals per hour of sleep. Awakenings were also included in the arousal index. The DESAT was calculated based on the total number of episodes of oxygen desaturation ( $\geq 3 \%$ drop in $\mathrm{S}_{\mathrm{p}} \mathrm{o}_{2}$ from baseline)/h of sleep. The lowest value of $\mathrm{S}_{\mathrm{O}_{2}}\left(\mathrm{~S}_{\mathrm{p}} \mathrm{O}_{2}\right.$ nadir) ${ }^{\mathrm{p}}$ was also recorded. $\mathrm{S}_{\mathrm{p}} \mathrm{o}_{2}$ falls caused by artifact were eliminated based on loss of the pulse wave form.

\section{Statistical Analysis}

All polysomnographic and metabolic parameters were first checked for normality and were log-transformed if found to be not normally distributed. The results were summarized as mean plus or minus the $\mathrm{SD}$, with the range disclosed. Each polysomnographic index was tested for correlation (Pearson product-moment correlation coefficient) with every metabolic parameter. Subsequently, those pairs that were found to be significantly correlated were subjected to further analysis by multiple linear regression, with the metabolic index as the outcome variable and the polysomnographic index as the key predictor variable. Indexes of adiposity (BMI, BMI z-score, total body fat, percentage body fat, trunk fat, or percentage trunk fat) and age were added as other predictor variables, to adjust for potential confounding influences on the metabolic parameter. Finally, the standardized regression coefficient $\beta$ (described further in the following section) between the metabolic and polysomnographic indexes, obtained from the multiple linear regression model, was calculated. A $P$ value of $\leq 0.05$ was considered to be statistically significant.

\section{Standardized Regression Coefficient $\beta$}

Multiple linear regression analysis provides a means to untangle the unique influence of each explanatory or independent variable on the outcome (37). The regression coefficients are a means to quantify the extent to which the different, albeit related, factors each uniquely contribute to the outcome. Due to the difference in units of each regression coefficient, they cannot be directly compared. However, by using standardized regression coefficients, which are unit-less, the 
relative strength of each explanatory variable on the outcome can be measured. The standardized regression coefficient $\beta$ for explanatory or independent variable $x_{i}$ is defined as $\beta_{i}=b_{i}\left(\mathrm{SD}_{i} / \mathrm{SD}_{i}\right)$, where $b_{i}$ is the regression coefficient for $x_{i}, \mathrm{SD}_{i}$ is the $\mathrm{SD}$ of $x_{i}$, and $\mathrm{SD}_{y}$ is the $\mathrm{SD}$ of the outcome or dependent variable $y(37)$.

\section{ACKNOWLEDGMENTS}

We thank the Children's Hospital Los Angeles General Clinical Research Center for provision of staff and funding for this project. We also thank Richard Bergman, Michael Goran, and staff for providing MINMOD software and expertise for interpretation of the FSIVGTT. We also acknowledge the subjects and their families for participating in this project.

\section{STATEMENT OF FINANCIAL SUPPORT}

This work was supported in part by National Institutes of Health $(\mathrm{NIH})$ grants HL090451 and EB001978; University of Southern California Center for Transdisciplinary Research on Energetics and Cancer grant TREC U54 CA 116848; and grant M01 RR00047 from Children's Hospital Los Angeles General Clinical Research Center, with funds provided by the National Center for Research Resources, NIH.

\section{REFERENCES}

1. Han JC, Lawlor DA, Kimm SY. Childhood obesity. Lancet 2010;375:1737-48

2. Ip MS, Lam B, Ng MM, Lam WK, Tsang KW, Lam KS. Obstructive sleep apnea is independently associated with insulin resistance. Am J Respir Crit Care Med 2002;165:670-6.

3. Tasali E, Ip MS. Obstructive sleep apnea and metabolic syndrome: alterations in glucose metabolism and inflammation. Proc Am Thorac Soc 2008;5:207-17.

4. Punjabi NM, Beamer BA. Alterations in Glucose Disposal in Sleep-disordered Breathing. Am J Respir Crit Care Med 2009;179:235-40.

5. Nock NL, Li L, Larkin EK, Patel SR, Redline S. Empirical evidence for "syndrome Z": a hierarchical 5-factor model of the metabolic syndrome incorporating sleep disturbance measures. Sleep 2009;32:615-22.

6. Lévy P, Bonsignore MR, Eckel J. Sleep, sleep-disordered breathing and metabolic consequences. Eur Respir J 2009;34:243-60.

7. de la Eva RC, Baur LA, Donaghue KC, Waters KA. Metabolic correlates with obstructive sleep apnea in obese subjects. J Pediatr 2002;140:654-59.

8. Verhulst SL, Schrauwen N, Haentjens D, et al. Sleep-disordered breathing and the metabolic syndrome in overweight and obese children and adolescents. J Pediatr 2007;150:608-12.

9. Tauman R, O'Brien LM, Ivanenko A, Gozal D. Obesity rather than severity of sleep-disordered breathing as the major determinant of insulin resistance and altered lipidemia in snoring children. Pediatrics 2005;116:e66-73.

10. Waters KA, Sitha S, O'Brien LM, et al. Follow-up on metabolic markers in children treated for obstructive sleep apnea. Am J Respir Crit Care Med 2006;174:455-60.

11. Shaibi GQ, Goran MI. Examining metabolic syndrome definitions in overweight Hispanic youth: a focus on insulin resistance. J Pediatr 2008;152:171-6.

12. Goran MI, Bergman RN, Cruz ML, Watanabe R. Insulin resistance and associated compensatory responses in african-american and Hispanic children. Diabetes Care 2002;25:2184-90.

13. Matthews DR, Hosker JP, Rudenski AS, Naylor BA, Treacher DF, Turner RC. Homeostasis model assessment: insulin resistance and beta-cell function from fasting plasma glucose and insulin concentrations in man. Diabetologia 1985;28:412-9.

14. Kahn SE. The importance of the beta-cell in the pathogenesis of type 2 diabetes mellitus. Am J Med 2000;108:Suppl 6a:2S-8S.

15. Bergman RN. Minimal model: perspective from 2005. Horm Res 2005;64:Suppl 3:8-15.
16. Verhulst SL, Rooman R, Van Gaal L, De Backer W, Desager K. Is sleepdisordered breathing an additional risk factor for the metabolic syndrome in obese children and adolescents? Int J Obes (Lond) 2009;33:8-13.

17. Flint J, Kothare SV, Zihlif M, et al. Association between inadequate sleep and insulin resistance in obese children. J Pediatr 2007;150:364-9.

18. Redline S, Storfer-Isser A, Rosen CL, et al. Association between metabolic syndrome and sleep-disordered breathing in adolescents. Am J Respir Crit Care Med 2007;176:401-8.

19. Li AM, Chan MH, Chan DF, et al. Insulin and obstructive sleep apnea in obese Chinese children. Pediatr Pulmonol 2006;41:1175-81.

20. Kaditis AG, Alexopoulos EI, Damani E, et al. Obstructive sleep-disordered breathing and fasting insulin levels in nonobese children. Pediatr Pulmonol 2005;40:515-23.

21. Dubern B, Tounian P, Medjadhi N, Maingot L, Girardet JP, Boulé M. Pulmonary function and sleep-related breathing disorders in severely obese children. Clin Nutr 2006;25:803-9.

22. Canapari CA, Hoppin AG, Kinane TB, Thomas BJ, Torriani M, Katz ES, Relationship between sleep apnea, fat distribution, and insulin resistance in obese children. J Clin Sleep Med 2011;7:268-73.

23. Stamatakis KA, Punjabi NM. Effects of sleep fragmentation on glucose metabolism in normal subjects. Chest 2010;137:95-101.

24. Späth-Schwalbe E, Gofferje M, Kern W, Born J, Fehm HL. Sleep disruption alters nocturnal ACTH and cortisol secretory patterns. Biol Psychiatry 1991;29:575-84.

25. Follenius M, Brandenberger G, Bandesapt JJ, Libert JP, Ehrhart J. Nocturnal cortisol release in relation to sleep structure. Sleep 1992;15:21-7.

26. Dinneen S, Alzaid A, Miles J, Rizza R. Metabolic effects of the nocturnal rise in cortisol on carbohydrate metabolism in normal humans. J Clin Invest 1993;92:2283-90.

27. Andrews RC, Walker BR. Glucocorticoids and insulin resistance: old hormones, new targets. Clin Sci 1999;96:513-23.

28. Iiyori N, Alonso LC, Li J, et al. Intermittent hypoxia causes insulin resistance in lean mice independent of autonomic activity. Am J Respir Crit Care Med 2007;175:851-7.

29. Kelly A, Dougherty S, Cucchiara A, Marcus CL, Brooks LJ. Catecholamines, adiponectin, and insulin resistance as measured by HOMA in children with obstructive sleep apnea. Sleep 2010;33:1185-91.

30. Kelly LA, Lane CJ, Weigensberg MJ, Toledo-Corral CM, Goran MI. Pubertal changes of insulin sensitivity, acute insulin response, and $B$-cell function in overweight Latino youth. J Pediatr 2011;158:442-6.

31. Bergman RN, Prager R, Volund A, Olefsky JM. Equivalence of the insulin sensitivity index in man derived by the minimal model method and the euglycemic glucose clamp. J Clin Invest 1987;79:790-800.

32. Boston RC, Stefanovski D, Moate PJ, Sumner AE, Watanabe RM, Bergman RN. MINMOD Millennium: a computer program to calculate glucose effectiveness and insulin sensitivity from the frequently sampled intravenous glucose tolerance test. Diabetes Technol Ther 2003;5:1003-15.

33. Bergman RN, Bowden CR. The minimal model approach to quantification of factors controlling glucose disposal in man. In: Cobelli C, Bergman RN, eds. Carbohydrate Metabolism. Chichester, UK: Wiley, 1981:269-296.

34. Katz A, Nambi SS, Mather K, et al. Quantitative insulin sensitivity check index: a simple, accurate method for assessing insulin sensitivity in humans. J Clin Endocrinol Metab 2000;85:2402-10.

35. Iber C, Ancoli-Israel S, Chesson A, Quan SF for the American Academy of Sleep Medicine. The AASM Manual for the Scoring of Sleep and Associated Events: Rules, Terminology and Technical Specification, 1st ed. Westchester, Illinois: American Academy of Sleep Medicine, 2007.

36. Grigg-Damberger M, Gozal D, Marcus CL, et al. The visual scoring of sleep and arousal in infants and children. J Clin Sleep Med 2007;3:201-40.

37. Hassard TH. Understanding Biostatistics. 3rd edn. St. Louis, MO: Mosby Year Book, 1991: 247-55. 